\title{
Entangled Light in Moving Frames
}

\author{
Attila J. Bergou, Robert M. Gingrich, and Christoph Adami \\ Quantum Computing Technologies Group, Jet Propulsion Laboratory 126-347 \\ California Institute of Technology, Pasadena, CA 91109-8099
}

(Dated: June 6, 2018)

\begin{abstract}
We calculate the entanglement between a pair of polarization-entangled photon beams as a function of the reference frame, in a fully relativistic framework. We find the transformation law for helicity basis states and show that, while it is frequency independent, a Lorentz transformation on a momentum-helicity eigenstate produces a momentum-dependent phase. This phase leads to changes in the reduced polarization density matrix, such that entanglement is either decreased or increased, depending on the boost direction, the rapidity, and the spread of the beam.
\end{abstract}

The second quantum revolution [1] is changing the ways in which we think about quantum systems. Rather than just describing and predicting their behavior, we now use new tools such as quantum information theory to organize and control quantum systems, and turn their non-classical features to our advantage in creating quantum technology. The central feature that makes quantum technology possible is quantum entanglement, which implies that particles or fields that have once interacted are connected by an overall wave function even if they are detected arbitrarily far away from each other. Such entangled pairs, first discussed after their introduction by Einstein, Podolsky, and Rosen [2], are crucial in technology such as quantum teleportation [3] and superdense coding [4]. Furthermore, quantum entanglement is critical in applications such as quantum optical interferometry, where quantum entangled $N$-photon pairs can increase the shot-noise limited sensitivity up to the Heisenberg limit [5].

While quantum entanglement as a resource has been studied extensively within the last decade [6], it was realized only recently that this resource is frame-dependent, and changes non-trivially under Lorentz transformations 17, 8, 9, 10, 11, 12]. In particular, Gingrich and Adami showed that the entanglement between the spins of a pair of massive spin- $1 / 2$ particles depends on the reference frame, and can either decrease or increase depending on the wave-function of the pair [11]. A consequence of this finding is that the entanglement resource could be manipulated by applying frame changes only. Many applications of quantum technology, however, involve entangled photons rather than massive spin- $1 / 2$ particles, to which the massive theory does not apply. In this letter, we work out the consequences of Lorentz transformations on photon beams that are entangled in polarization. Each photon beam is described by a Gaussian wave packet with a particular angular spread in momentum, and for the sake of being definite we discuss a state whose polarization entanglement can be thought of as being produced by down-conversion. Because both spin- $1 / 2$ particles and photons can be used as quantum information carriers (qubits), the present calculation also contributes to the nascent field of Relativistic Quantum
Information Theory $[13]$.

In order to calculate how a polarization-entangled photon state transforms under Lorentz transformations, we need to discuss the behavior of the photon basis sates. Because there is no rest frame for a massless particle, the analysis of the spin (polarization) properties is quite distinct from the massive case. For instance, instead of using $p^{\mu}=(m, \mathbf{0})$ as the standard 4-vector (see [1] ), we have to define the massless analog $k^{\mu}=(1, \hat{\mathbf{z}})$. Note that $k^{\mu}$ has no parameter $m$ and is no longer invariant under all rotations. In fact, the little group of $k^{\mu}$ is isomorphic to the non-compact two-dimensional Euclidean group $E(2)$ (the set of transformations that map a twodimensional Euclidean plane onto itself) . For a massless spin-one particle the standard vector allows us to define the eigenstate

$$
\begin{aligned}
P^{\mu}|\hat{\mathbf{z}} \lambda\rangle & =k^{\mu}|\hat{\mathbf{z}} \lambda\rangle \\
J_{z}|\hat{\mathbf{z}} \lambda\rangle & =\lambda|\hat{\mathbf{z}} \lambda\rangle,
\end{aligned}
$$

where $\hat{\mathbf{z}}$ is a unit vector pointing in the z-direction. Since the particle is massless, $\lambda$ is restricted to \pm 1 [14].

The momentum-helicity eigenstates are defined as

$$
|\mathbf{p} \lambda\rangle=H(\mathbf{p})|\hat{\mathbf{z}} \lambda\rangle,
$$

where $H(\mathbf{p})$ is a Lorentz transformation that takes $\hat{\mathbf{z}}$ to p. The choice of $H(\mathbf{p})$ is not unique, and different choices lead to different interpretations of the parameter $\lambda$. For instance, in the massive case the choice of $H(\mathbf{p})$ can lead to $\lambda$ being either the rest frame spin or the helicity. In the present case it is convenient to choose

$$
H(\mathbf{p})=R(\hat{\mathbf{p}}) L_{z}\left(\xi_{\mathbf{p}}\right),
$$

where $L_{z}\left(\xi_{\mathbf{p}}\right)$ is a Lorentz boost along $\hat{\mathbf{z}}$ that takes $\hat{\mathbf{z}}$ to $|\mathbf{p}| \hat{\mathbf{z}}$ and $R(\mathbf{p})$ is a rotation that takes $\hat{\mathbf{z}}$ to $\hat{\mathbf{p}}$, while $\xi_{\mathbf{p}}$ is the rapidity of the moving frame,

$$
\xi_{\mathbf{p}}=\ln |\mathbf{p}| \text {. }
$$

For a parameterization in polar coordinates, we can write $\hat{\mathbf{p}}=(\sin \theta \cos \phi, \sin \theta \sin \phi, \cos \theta)$

$$
R(\hat{\mathbf{p}}) \equiv R_{z}(\phi) R_{y}(\theta) .
$$


Again, this choice of $R(\hat{\mathbf{p}})$ is not unique (see for example [17) but particularly easy to deal with in this context. An arbitrary two-particle state in this formalism can be written as

$$
\left|\Psi_{A A^{\prime} B B^{\prime}}\right\rangle=\iint \sum_{\lambda \sigma} g_{\lambda \sigma}(\mathbf{p}, \mathbf{q})|\mathbf{p} \lambda\rangle_{A A^{\prime}}|\mathbf{q} \sigma\rangle_{B B^{\prime}} \tilde{\mathrm{d}} \mathbf{p} \tilde{\mathrm{d}} \mathbf{q},
$$

where $|\mathbf{p} \lambda\rangle_{A A^{\prime}}$ and $|\mathbf{q} \sigma\rangle_{B B^{\prime}}$ correspond to the momentum and helicity states, as defined in Eq. (3), of photons $A$ and $B$. Furthermore, $\tilde{d} \mathbf{p}$ and $\tilde{d} \mathbf{q}$ are the Lorentz-invariant momentum integration measures:

$$
\widetilde{\mathrm{d}} \mathbf{p} \equiv \frac{\mathrm{d}^{3} \mathbf{p}}{2|\mathbf{p}|}
$$

and the functions $g_{\lambda \sigma}(\mathbf{p}, \mathbf{q})$ must satisfy

$$
\iint \sum_{\lambda \sigma}\left|g_{\lambda \sigma}(\mathbf{p}, \mathbf{q})\right|^{2} \widetilde{\mathrm{d}} \mathbf{p} \widetilde{\mathrm{d}} \mathbf{q}=1 .
$$

To work out how a Lorentz boost affects an entangled state, we must understand how the basis states $|\mathbf{p} \lambda\rangle$ transform. Following [14, 15], we apply a boost $\Lambda$ to $|\mathbf{p} \lambda\rangle$

$$
\Lambda|\mathbf{p} \lambda\rangle=H(\Lambda \mathbf{p}) H(\Lambda \mathbf{p})^{-1} \Lambda H(\mathbf{p})|\hat{\mathbf{z}} \lambda\rangle,
$$

where $H(\Lambda \mathbf{p})^{-1} \Lambda H(\mathbf{p})$ is a member of the little group of $\hat{\mathbf{z}}$ (leaves $\hat{\mathbf{z}}$ invariant), and hence is a rotation and/or translation in the $x, y$ plane. The translations can be shown 14 not to affect the spin/helicity, and we are thus left with just a rotation by an angle $\Theta(\Lambda, \mathbf{p})$. Using the parameterization $\mathbf{p}=p(\sin \theta \cos \phi, \sin \theta \sin \phi, \cos \theta)$ and solving for $\Theta(\Lambda, \mathbf{p})$ we obtain

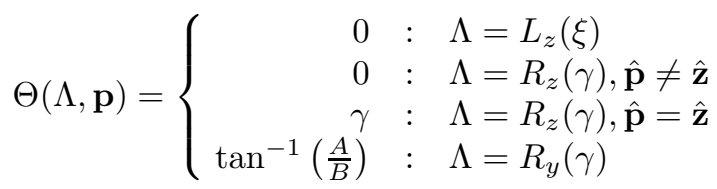

for different Lorentz transformations and momenta, where

$$
\begin{aligned}
& A=\sin \gamma \sin \theta \\
& B=\cos \theta \sin \gamma \cos \phi+\cos \gamma \sin \phi .
\end{aligned}
$$

Noting that

$$
R_{z}\left(\Theta\left(\Lambda^{\prime} \Lambda, \mathbf{p}\right)\right)=R_{z}\left(\Theta\left(\Lambda^{\prime}, \Lambda \mathbf{p}\right)\right) R_{z}(\Theta(\Lambda, \mathbf{p}))
$$

and taking advantage of the fact that all Lorentz boosts can be constructed using $L_{z}, R_{z}$ and $R_{y}$, Eq. (11) allows us to find $\Theta(\Lambda, \mathbf{p})$ for any $\Lambda$, and any momentum p. Applying this rotation to the momentum-helicity eigenstate of a massless particle we obtain

$$
\Lambda|\mathbf{p} \lambda\rangle=\mathrm{e}^{-\mathrm{i} \lambda \Theta(\Lambda, \mathbf{p})}|\Lambda \mathbf{p} \lambda\rangle .
$$

Typically, it is the polarization of a photon that is measured in quantum optics experiments, not the helicity. Let us therefore examine the effects of a Lorentz transformation on a photon's polarization 4 -vector.

The polarization 4 -vectors for positive and negative helicity states are given by

$$
\epsilon_{ \pm}^{\mu}(\hat{\mathbf{p}})=\frac{R(\hat{\mathbf{p}})}{\sqrt{2}}\left[\begin{array}{c}
0 \\
1 \\
\pm i \\
0
\end{array}\right]
$$

A general polarization vector is, of course, formed by the superposition of the two basis vectors. According to 9, 18], for a given 4-momentum $p^{\mu}$ and associated polarization $\epsilon^{\mu}$, a Lorentz boost has the following effect:

$$
D(\Lambda) \epsilon^{\mu}=R(\Lambda \hat{\mathbf{p}}) R(\hat{\mathbf{p}})^{-1} \epsilon^{\mu} .
$$

However, this transformation is only correct for pure boosts in the z-direction, or rotations around the z-axis if this axis is not the momentum axis (as for those cases the angle $\Theta(\Lambda, \mathbf{p})$ in Eq. (11) vanishes). In general, the four-vector $\epsilon^{\mu}$ transforms as

$$
D(\Lambda) \epsilon^{\mu}=R(\Lambda \hat{\mathbf{p}}) R_{z}(\Theta(\Lambda, \mathbf{p})) R(\hat{\mathbf{p}})^{-1} \epsilon^{\mu} .
$$

It is helpful to write $D(\Lambda)$ in an alternative form

$$
D(\Lambda) \epsilon^{\mu}=\Lambda \epsilon^{\mu}-\frac{\left(\Lambda \epsilon^{\mu}\right)^{0}}{\left(\Lambda p^{\mu}\right)^{0}} \Lambda p^{\mu},
$$

where $\left(\Lambda \epsilon^{\mu}\right)^{0}$ and $\left(\Lambda p^{\mu}\right)^{0}$ denote the time-like component of the transformed polarization and momentum 4vectors, respectively. The form Eq. (19) agrees with the general law described in [16]. The proof that Eqs. (18) and (19) are equivalent is non-trivial, but an outline is as follows. Note that both forms of $D(\Lambda)$ obey

$$
D\left(\Lambda^{\prime}\right) D(\Lambda) \epsilon^{\mu}=D\left(\Lambda^{\prime} \Lambda\right) \epsilon^{\mu}
$$

and both forms have the property

$$
D(R) \epsilon^{\mu}=R \epsilon^{\mu}
$$

where $R$ is a rotation. An explicit calculation of $D\left(L_{z}(\xi)\right)$ then shows that they are equivalent.

The second term on the right hand side of Eq. (19) is just a momentum-dependent gauge transformation. It must be different for each momentum in order to keep a consistent overall (Coulomb) gauge. To see that this term leads to measurable consequences consider the polarization vector for classical electromagnetic waves. The polarization vector points along the gauge-invariant electric field, and the direction of this vector undergoes the same transformation as in Eq. (19) (or 18) when acted on by a Lorentz transformation. In fact, the magnitude of the electric field undergoes the same transformation 
as the diameter of an infinitesimal circle centered at the momentum. This holds for any Lorentz transformation and momentum. A detailed study of this transformation will be published elsewhere.

In the following, we investigate two entangled photon beams moving along the z-axis. The beams are in a momentum product state, and fully entangled in polarization,

$$
g_{\lambda \sigma}(\mathbf{p}, \mathbf{q})=\frac{1}{\sqrt{2}} \delta_{\lambda \sigma} \mathrm{e}^{\mathrm{i} \lambda \phi_{\mathbf{p}}} \mathrm{e}^{\mathrm{i} \sigma \phi_{\mathbf{q}}} f(\mathbf{p}) f(\mathbf{q}) .
$$

In Eq. (22), $\phi_{\mathbf{p}}$ and $\phi_{\mathbf{q}}$ are the polar angles of $\mathbf{p}$ and $\mathbf{q}$ respectively. The phase factors $\mathrm{e}^{\mathrm{i} \lambda \phi_{\mathbf{p}}} \mathrm{e}^{\mathrm{i} \sigma \phi_{\mathbf{q}}}$ allow us to write the state as

$$
|\Psi\rangle=\iint \frac{1}{\sqrt{2}}\left(\left|h_{\mathbf{p}}\right\rangle\left|h_{\mathbf{q}}\right\rangle-\left|v_{\mathbf{p}}\right\rangle\left|v_{\mathbf{q}}\right\rangle\right) f(\mathbf{p})|\mathbf{p}\rangle f(\mathbf{q})|\mathbf{q}\rangle \tilde{d} \mathbf{p} \tilde{d} \mathbf{q},
$$

where $\left|h_{\mathbf{p}}\right\rangle$ and $\left|v_{\mathbf{p}}\right\rangle$ are approximations of horizontal and vertical polarization given by [19]

$$
\begin{array}{r}
\left|h_{\mathbf{p}}\right\rangle \equiv \frac{1}{\sqrt{2}}\left(\mathrm{e}^{\mathrm{i} \phi_{\mathbf{p}}} \epsilon_{+}^{\mu}(\hat{\mathbf{p}})+\mathrm{e}^{-\mathrm{i} \phi_{\mathbf{p}}} \epsilon_{-}^{\mu}(\hat{\mathbf{p}})\right) \\
\left|v_{\mathbf{p}}\right\rangle \equiv \frac{-\mathrm{i}}{\sqrt{2}}\left(\mathrm{e}^{\mathrm{i} \phi_{\mathbf{p}}} \epsilon_{+}^{\mu}(\hat{\mathbf{p}})-\mathrm{e}^{-\mathrm{i} \phi_{\mathbf{p}}} \epsilon_{-}^{\mu}(\hat{\mathbf{p}})\right) .
\end{array}
$$

So, for small $\theta$ (small azimuthal spread of the momentum distribution) we have:

$$
\begin{aligned}
\left|h_{\mathbf{p}}\right\rangle & \simeq \hat{\mathbf{x}} \\
\left|v_{\mathbf{p}}\right\rangle & \simeq \hat{\mathbf{y}},
\end{aligned}
$$

and Eq. 23) is a close approximation to a polarization Bell state. Omitting the phase factors in (22) and (24) instead describes a photon beam where horizontal and vertical polarizations point in the $\hat{r}$ and $\hat{\phi}$ directions, respectively (see Fig. 1).

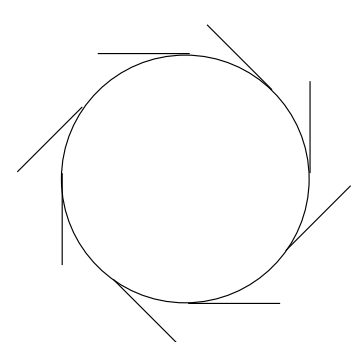

(a)

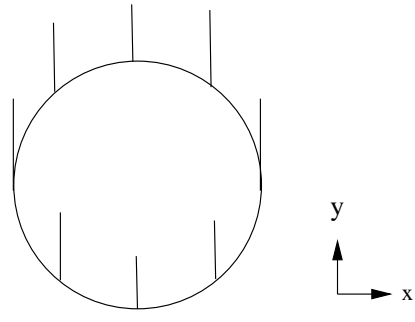

(b)
FIG. 1: (a): "Standard" vertical polarization vectors $\left|v_{\mathbf{p}}^{\prime}\right\rangle$ point in polar directions. (b): "True" vertical polarization vectors $\left|v_{\mathbf{p}}\right\rangle$ remain mostly in the $\mathrm{x}-\mathrm{y}$ plane.

We specifically consider the beams to have a Gaussian spread in the azimuthal direction,

$$
f(\mathbf{p})=\frac{1}{N(\sigma)} \exp \left(-\frac{1}{2}\left(\frac{\theta}{\sigma_{\theta}}\right)^{2}\right) \delta\left(|\mathbf{p}|-p_{0}\right),
$$

where $\sigma_{\theta}$ is a parameter which controls the spread of the beam, $\theta$ is the azimuthal angle of the momentum vector, and $p_{0}$ is the magnitude of the momentum of the photon beam, which we arbitrarily set to unity. We do not take into account a spread in the magnitude of the momentum because the magnitude, $\omega$, is just a constant multiplying the momentum 4 -vector and so

$$
\begin{aligned}
\Lambda p^{\mu} & =\Lambda(\omega, \omega \hat{\mathbf{p}}) \\
& =\Lambda \omega(1, \hat{\mathbf{p}}) \\
& =\omega \Lambda(1, \hat{\mathbf{p}}) .
\end{aligned}
$$

Inserting this result into Eq. (19), we see that the $\omega$ dependence cancels.

We now boost the state Eq. (23) and trace out the momentum degrees of freedom to construct the polarization density matrix. Because photons are spin-one particles, they constitute three-level systems (even though they are constrained to be transverse for any particular momentum). In order to calculate the entanglement present in the quantum state, we therefore cannot use Wootters' concurrence [20], as it is only a measure of entanglement for two-state quantum entangled systems. Instead, we use here "log negativity", an entanglement measure introduced by Vidal and Werner [21]. This measure is defined as

$$
L N(\rho)=\log _{2}\left\|\rho^{T_{A}}\right\|,
$$

where $\|\rho\|$ is the trace norm and $\rho^{T_{A}}$ is the partial transpose of $\rho . L N(\rho)$ is a measure of the entanglement but is unable to detect bound entanglement. We can now calculate the change in log negativity explicitly for a Lorentz boost with rapidity $\xi$ at an angle $\alpha$ with respect to the photon momentum, i.e., a Lorentz transformation

$$
\Lambda=R_{y}(\alpha) L_{z}(\xi) R_{y}(\alpha)^{-1},
$$

applied to Eq. (23). Fig. 2] summarizes the results of varying the boost direction, $\alpha$, for a given spread, $\sigma_{\theta}$, and shows that the entanglement can increase or decrease, depending on boost direction. For $\alpha=0$, positive $\xi$ corresponds to boosting the photon in the direction of the detector. Note that the entanglement at zero rapidity is only about half its maximal value, because the angular spread of the momentum leaves the spin degrees of freedom in a mixed state after tracing out momentum. In general, boosts in the direction of motion tend to increase the entanglement to saturation, while boosts away from it decrease it. As $\alpha$ approaches $\frac{\pi}{2}$, the effect on entanglement becomes symmetric.

Fig. 3 summarizes the effect of applying the boost in Eq. (31) for varying spreads in the momentum distribution, for a boost direction given by $\alpha=2 \pi / 5$. Distributions with small spread, $\sigma_{\theta} \leq 0.1$, tend to change entanglement only imperceptively, while for larger spread the entanglement changes become more pronounced. 


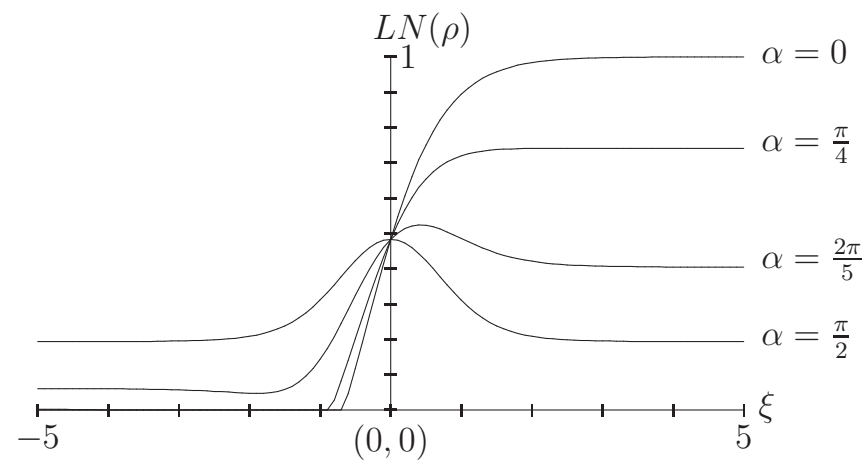

FIG. 2: Log negativity of the spin as a function of rapidity shown for various boost directions. $\alpha$ is the polar angle of the boost direction. For all of the curves the angular spread is the same, $\sigma=1.0$.

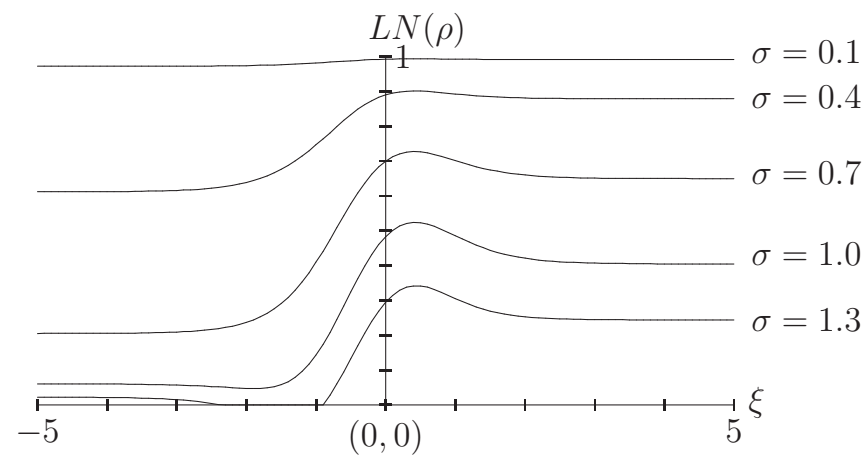

FIG. 3: Log negativity as a function of rapidity shown for beams of various angular spreads, $\sigma$. For all of the above curves the boost direction $\alpha=\frac{2 \pi}{5}$.

Note that for $\sigma_{\theta}=1.3$ the entanglement becomes zero (for boosts of negative rapidity) and then increases. This appears to happen because the momentum spread becomes so large that a significant portion of the beam is in fact moving in the $-\hat{\mathbf{z}}$ direction. Because of the collimating effect that a Lorentz boost has on the beam, the entanglement can actually increase in such a situation.

We have derived the relativistic transformation law for photon polarizations, and shown that the entanglement of polarization-entangled pairs of photon beams depends on the reference frame. Boosting a detector (even at an angle) towards the beams increases this entanglement because the momentum distribution is shrunk by the boost (see also 12]). The type of entangled beams that we have investigated in this letter are idealizations of realistic states that can be created using parametric down-conversion. In principle, therefore, the effects discussed here should become relevant as soon as linearoptics based quantum technology is created that is placed on systems that move with respect to a detector (or when the detector moves with respect to such a system).

We would like to thank Jonathan Dowling, and the members of the JPL Quantum Computing Group, for useful discussions and encouragement. We also acknowl- edge János Bergou for helpful suggestions. This work was carried out at the Jet Propulsion Laboratory (California Institute of Technology) under a contract with the $\mathrm{Na}$ tional Aeronautics and Space Administration, with support from the National Security Agency, the Advanced Research and Development Activity, the Defense Advanced Research Projects Agency, the National Reconnaissance Office, and the Office of Naval Research.

[1] J.P. Dowling and G. J. Milburn, Phil. Trans. Roy. Soc. London 361 (2003), to appear.

[2] A. Einstein, B. Podolsky, and N. Rosen, Phys. Rev. 47, 777-780 (1935).

[3] C.H. Bennett et al., Phys. Rev. Lett. 70, 1895(1993).

[4] C.H. Bennett and S.J. Wiesner, Phys. Rev. Lett. 69, 2881 (1992).

[5] J.P. Dowling, Phys. Rev. A 57, 4736 (1998).

[6] M.A. Nielsen and I.L. Chuang. Quantum Computation and Quantum Communication (Cambridge University Press, 2000).

[7] A. Peres, P.F. Scudo, and D.R. Terno, Phys. Rev. Lett. 88, 230402 (2002).

[8] M. Czachor, Phys. Rev. A 55, 72 (1997).

[9] P.A. Alsing and G.J. Milburn, Quantum Inf. Comput. 2, 487 (2002).

[10] H. Terashima and M. Ueda, Int. J. Quant. Inf. 1 (2003).

[11] R.M. Gingrich and C. Adami, Phys. Rev. Lett. 89, 270402 (2002).

[12] A. Peres and D.R. Terno, quant-ph/0208128

[13] A. Peres and D.R. Terno, Rev. Mod. Phys. 75 (2003), to appear.

[14] E.P. Wigner, Ann. of Math. 40, 149-204 (1939).

[15] W.K. Tung, Group Theory in Physics (World Scientific Publishing, 1985).

[16] S. Weinberg, The Quantum Theory of Fields, Vol. I (Cambridge University Press, 1995).

[17] F.J. Yndurain, Relativistic Quantum Mechanics and Introduction to Field Theory (Springer Verlag, 1996).

[18] D. Han, Y.S.Kim and D. Son, Phys. Rev. D 31, 328-330 (1985).

[19] The choice of basis vectors $\left|h_{\mathbf{p}}\right\rangle$ and $\left|v_{\mathbf{p}}\right\rangle$ that guarantees polarization vectors as close as possible to the laboratory $\hat{\mathbf{x}}, \hat{\mathbf{y}}$ directions is obtained instead by using

$$
\phi_{\mathbf{p}} \rightarrow \psi_{\mathbf{p}}
$$

where $\psi_{\mathbf{p}}$ is such that

$$
\tan \left(2 \psi_{\mathbf{p}}\right)=\tan (2 \phi) \frac{2 \cos (\theta)}{1+\cos ^{2}(\theta)} .
$$

However, we used $\phi_{\mathbf{p}}$ in the present calculations because it is approximately equal to $\psi_{\mathbf{p}}$ except when $\theta$ is large, and much more convienient for our numerical simulations. We carried out a subset of the calculations displayed in Figs. 2 and 3 using the optimal angle $\psi_{\mathbf{p}}$, and found essentially unchanged results.

[20] W.K. Wootters, Phys. Rev. Lett. 80, 2245 (1998).

[21] G. Vidal and R.F. Werner, Phys. Rev. A 65, 032314 (2002). 